\title{
Mixed-phase description of colossal magnetoresistive manganites
}

\author{
Alexander Weiße, ${ }^{1}$ Jan Loos, ${ }^{2}$ and Holger Fehske $^{3}$ \\ ${ }^{1}$ Physikalisches Institut, Universität Bayreuth, 95440 Bayreuth, Germany \\ ${ }^{2}$ Institute of Physics, Czech Academy of Sciences, 16200 Prague, Czech Republic \\ ${ }^{3}$ Institut für Physik, Ernst-Moritz-Arndt Universität Greifswald, 17487 Greifswald, Germany
}

(Dated: September 10, 2018)

\begin{abstract}
In view of recent experiments, indicating the spatial coexistence of conducting and insulating regions in the ferromagnetic metallic phase of doped manganites, we propose a refined mixedphase description. The model is based on the competition of a double-exchange driven metallic component and a polaronic insulating component, whose volume fractions and carrier concentrations are determined self-consistently by requiring equal pressure and chemical potential. The resulting phase diagram as well as the order of the phase transition are in very good agreement with measured data. In addition, modelling the resistivity of the mixed, percolative phase by a random resistor network, we obtain a pronounced negative magnetoresistance in the vicinity of the Curie temperature $T_{C}$.

PACS numbers: 71.10.-w, 75.47.Gk, 71.38.Ht, 71.30.+h
\end{abstract}

\section{MOTIVATION}

The peculiar properties of the ferromagnetic metallic phase of mixed-valence manganites (e.g. $\mathrm{La}_{1-x} \mathrm{Ca}_{x} \mathrm{MnO}_{3}$ with $0.2 \lesssim x \leq 0.5$ ), in particular the large negative magnetoresistance close to the Curie temperature $T_{C}$, have been the subject of intense research activity over the last years $1,2,3,4$. There is now compelling experimental evidence, that the complex interplay of the electronic degrees of freedom (charge, spin and orbital) and the lattice leads to the spatial coexistence of regions with different properties rather than to the formation of a single homogeneous phase. Above $T_{C}$ the activated behaviour of the conductivity ${ }^{5.6 .7}$, the Hall conduction ${ }^{8}$ and the thermopower ${ }^{5.6}$, as well as the structure of the pair distribution function $(\mathrm{PDF})^{\frac{9}{}}$ indicate the formation of small polarons, i.e., of almost localised carriers within a surrounding lattice distortion. Interestingly these polarons continue to exist in the metallic phase below $T_{C}$, merely their volume fraction is noticeable reduced. Corresponding evidence is found in conductivity measurements ${ }^{10,11}$, muon spin relaxation ${ }^{12.13}$, high-resolution x-ray diffraction ${ }^{14}$, pulsed neutron diffraction ${ }^{15}$ and in x-ray absorption fine structure spectra ${ }^{16}$. Based on these experimental observations different scenarios for the coexistence of conducting and insulating regions within the metallic phase of the manganites were discussed, which relate the metalinsulator transition to phase separation ${ }^{4}$ and percolative phenomena ${ }^{17,18,19,20}$. In particular microscopic imaging techniques, like scanning tunneling spectroscopy ${ }^{21,22}$ or dark-field imaging 23 , seem to support the latter idea. However, as yet the detailed nature of the spatially coexisting regions or phases is not known very precisely and even the data for the corresponding length scales is contradictory 24,25 .

In a recent work ${ }^{26}$ we addressed the problem of coexisting conducting and insulating regions within the metallic phase of the manganites and proposed a phenomenological mixed-phase description, which is based on the competition of a polaronic insulating phase and a metallic, double-exchange driven ferromagnetic phase. Both phases are assumed to have an equal density of charge carriers and the percolative coexistence is accounted for by a metallic bandwidth, which depends on the volume fractions of the two components. The model is able to describe a finite polaronic volume fraction well below $T_{C}$ and yields rather realistic $x$ - $T$-phase diagrams. However, its sensitivity to external magnetic fields is much too weak and we made no attempt to describe resistivities or the large magnetoresistance.

Due to the insufficient knowledge of the two different components of the low temperature phase, the assumptions of our previous model may be problematic, in particular the balance of the two components could follow from different equilibrium conditions. In the present work we discard the condition of equal charge density within the polaronic insulating and the ferromagnetic metallic regions and require equal pressure instead. This approach is well justified, since one of the components is insulating and the length scale of the coexistence seems to be short enough, as to avoid long range Coulomb effects. We complete this new mixed-phase description by a model for the resistivity which is based on a random resistor network that accounts for the percolative nature of the low temperature phase. The phase diagram we obtain from the improved model is comparable to the previous results, however, the sensitivity to external magnetic fields is much stronger and the ansatz for the conductivity yields a rather large magnetoresistance close to $T_{C}$. In addition, the order of the phase transition from the ferromagnetic metallic to the paramagnetic insulating phase depends on the model parameters and in particular on the doping $x$, a feature which was observed for the real materials 27,28 . 


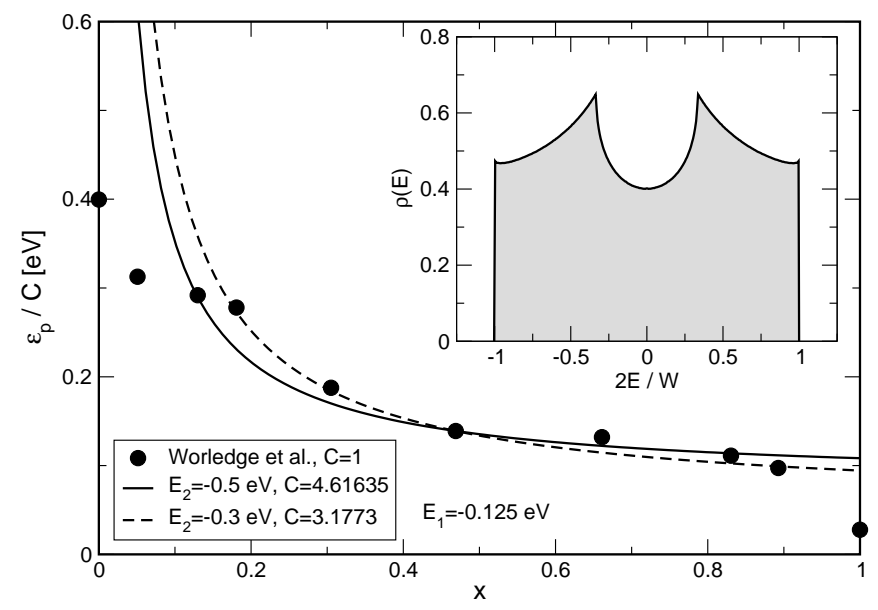

FIG. 1: Doping dependence of the polaronic binding energy: we compare experimental data ${ }^{7}$ from conductivity measurements (dots) with the $\epsilon_{p}$ from our ansatz (lines). Inset: Density of states used for the ferromagnetic metallic component.

\section{COEXISTING COMPONENTS}

In the doping range $0.2 \lesssim x \leq 0.5$ the electronic properties of the manganites are dominated by the well known double-exchange interaction ${ }^{29.30 .31}$ and the electron-lattice coupling. As long as the charge carriers (Mn $e_{g}$ holes) are mobile they mediate a ferromagnetic interaction between the localised $S=3 / 2$ spins formed by the $t_{2 g}$ electrons of the manganese. If the electron lattice interaction is strong enough small polarons can arise and the effective mass of the holes is increased by the lattice distortion accompanying its motion through the crystal. Due to this reduction of the mobility the ferromagnetic double-exchange may break down completely, but, as indicated by the mentioned experiments, this happens in a spatially inhomogeneous way. We model this feature by assuming a coexistence of a ferromagnetic metallic volume fraction, described mainly by the mean-field theory of double-exchange by Kubo and Ohata ${ }^{31}$, and of a polaronic insulating volume fraction, described by an exponentially narrow band and a paramagnetic spin background.

\section{A. Ferromagnetic metallic component}

Within mean-field theory the quantum doubleexchange matrix element ${ }^{30} \tilde{t}=t\left(S_{T}+1 / 2\right) /(2 S+1)$ for the hopping of an itinerant charge carrier between two neighbouring sites is averaged over all amplitudes and directions of the total bond spin $S_{T}$, which is assumed to be placed in an inner Weiss field $\lambda=\beta g \mu_{B} H_{\text {eff }}^{z}$ and an optional external magnetic field $\lambda^{\mathrm{ext}}=\beta g \mu_{B} H_{\text {ext }}^{z}$. Omitting orbital degrees of freedom the resulting Hamiltonian describes free fermions in an effective band of width

$$
W=\gamma_{S}\left[S\left(\lambda+\lambda^{\mathrm{ext}}\right)\right] W_{0},
$$

where $W_{0}$ denotes the bare band width and the field dependent prefactor is given by ${ }^{31}$

$$
\gamma_{S}[z]=\frac{S+1}{2 S+1}+\frac{S}{2 S+1} \operatorname{coth}\left(\frac{S+1}{S} z\right) B_{S}[z]
$$

with the Brillouin function

$$
B_{S}[z]=\frac{1}{2 S}\left[(2 S+1) \operatorname{coth} \frac{(2 S+1) z}{2 S}-\operatorname{coth} \frac{z}{2 S}\right] .
$$

To improve the above approximation of Kubo and Ohata 31 , we account for the orbitally anisotropic hopping ${ }^{32.33}$, which follows from the perovskite structure,

$$
t_{\alpha \beta}^{x / y}=\frac{t}{4}\left[\begin{array}{cc}
1 & \mp \sqrt{3} \\
\mp \sqrt{3} & 3
\end{array}\right], t_{\alpha \beta}^{z}=t\left[\begin{array}{ll}
1 & 0 \\
0 & 0
\end{array}\right], \alpha, \beta \in\{\theta, \varepsilon\}
$$

and for the strong on-site Coulomb interaction. In a mean-field sense both is achieved ${ }^{26}$ by working with the averaged density of states

$$
\varrho(E)=\frac{1}{2}\left(\varrho_{+}(E)+\varrho_{-}(E)\right),
$$

shown in the inset of Figure 1 The densities $\varrho_{\zeta}(E)$ belong to the two bands $(\zeta= \pm 1)$,

$$
\epsilon_{\mathbf{k}, \zeta}=-t\left(\sum_{\delta} \cos k_{\delta}+\zeta \sqrt{\frac{1}{4} \sum_{\delta, \delta^{\prime}}\left(\cos k_{\delta}-\cos k_{\delta^{\prime}}\right)^{2}}\right),
$$

resulting from nearest neighbour matrix elements of Equation (4) with $t=W / 6$, Equation (1).

We assume this non-interacting fermion model to be valid within the ferromagnetic volume fraction

$$
p^{(f)}=\frac{N^{(f)}}{N}
$$

of the sample. Of course $p^{(f)}$ is temperature and doping dependent. Its actual value is determined selfconsistently through the equations given in Section [II

\section{B. Polaronic insulating component}

In the remaining, polaronic part of the sample,

$$
p^{(p)}=\frac{N^{(p)}}{N}=1-p^{(f)},
$$

we assume all charge carriers to be self-trapped small polarons, i.e., their kinetic energy is exponentially suppressed and the band centre is shifted by a polaron binding energy $\epsilon_{p}$. To keep our model as simple as possible, we completely neglect the polaronic band width and consider only a dispersion-less level located at $\epsilon_{p}$. In our previous 
work $^{26}$, using certain energy arguments, we motivated a doping dependence of $\epsilon_{p}$ of the form

$$
\epsilon_{p}=\left(x^{-1}-1\right) E_{1}+E_{2} .
$$

Here $E_{1}$ and $E_{2}$ are effective model parameters describing the anti-Jahn-Teller effect and the usual polaron binding energy, respectively. If we discard the condition of equally charged metallic and insulating volume fractions, in a strict sense the old derivation is no longer valid. However, the comparison of the ansatz in Equation (9) with experimental data ${ }^{7}$ for the polaronic binding energy, which can be extracted from the temperature and doping dependence of the resistivity in the high-temperature paramagnetic phase, yields surprisingly good agreement over a wide doping range. Figure 1 illustrates that with our choice of parameters $\left\{E_{1}, E_{2}\right\}$ the functional form of $\epsilon_{p}$ matches the real data quite well, as long as $x$ is not too small. In absolute values the curves disagree by a constant factor of the order 3 to 5 , which is sufficient for a mean-field type theory.

\section{THERMODYNAMIC STABILITY CONDITIONS}

Based on the above assumptions for the two coexisting components of the low-temperature phase of the manganites we are now in the position to formulate equilibrium conditions. The essential change to our previous work concerns the assumption of equal pressure for carriers in the metallic and the insulating region, where the pressures are obtained from

$$
\begin{aligned}
& \pi^{(f)}=\frac{1}{\beta} \int \varrho(E) \log \left(1+e^{\beta(\mu-E)}\right) d E, \\
& \pi^{(p)}=\frac{1}{\beta} \int \delta\left(E-\epsilon_{p}\right) \log \left(1+e^{\beta(\mu-E)}\right) d E,
\end{aligned}
$$

with $\beta=1 /\left(k_{B} T\right)$. For each value of the inner field $\lambda$ the equation

$$
\pi^{(f)}=\pi^{(p)}=: \pi_{\text {eq }}
$$

then defines the chemical potential $\mu$, which is required to be equal for both components. Given $\mu$ the resulting carrier concentrations

$$
\begin{aligned}
& x^{(f)}=\int \frac{\varrho(E)}{e^{\beta(E-\mu)}+1} d E \\
& x^{(p)}=\int \frac{\delta\left(E-\epsilon_{p}\right)}{e^{\beta(E-\mu)}+1} d E
\end{aligned}
$$

in the coexisting regions define the two volume fractions by the equations

$$
x=p^{(f)} x^{(f)}+p^{(p)} x^{(p)} \quad \text { and } \quad p^{(f)}+p^{(p)}=1,
$$

which ensure the correct overall doping. Based on this set of equations we are able to calculate the free energy per site for the whole system,

$$
f=x \mu-\pi_{\mathrm{eq}}+f^{(s)},
$$

where

$$
\begin{aligned}
f^{(s)}=\frac{1}{\beta} & \left\{x \left[p ^ { ( f ) } \left(\lambda S B_{S}\left[S\left(\lambda+\lambda^{\mathrm{ext}}\right)\right]\right.\right.\right. \\
& \left.\left.-\log \nu_{S}\left[S\left(\lambda+\lambda^{\mathrm{ext}}\right)\right]\right)-p^{(p)} \log \nu_{S}\left[S \lambda^{\mathrm{ext}}\right]\right] \\
+ & (1-x)\left[p ^ { ( f ) } \left(\lambda \bar{S} B_{\bar{S}}\left[\bar{S}\left(\lambda+\lambda^{\mathrm{ext}}\right)\right]\right.\right. \\
& \left.\left.\left.-\log \nu_{\bar{S}}\left[\bar{S}\left(\lambda+\lambda^{\mathrm{ext}}\right)\right]\right)-p^{(p)} \log \nu_{\bar{S}}\left[\bar{S} \lambda^{\mathrm{ext}}\right]\right]\right\}
\end{aligned}
$$

denotes the spin part of the free energy $\left(\bar{S}=S+\frac{1}{2}=2\right)$ and

$$
\nu_{S}[z]=\sinh (z) \operatorname{coth}\left(\frac{z}{2 S}\right)+\cosh (z)
$$

is the spin partition function.

The set of equilibrium conditions is closed by the requirement that $\lambda$ is chosen such that the free energy is minimal. The same is required, if the above equations have more than a single solution. A finite value of $\lambda$ corresponds to a ferromagnetically ordered metallic component, and if in addition the corresponding volume fraction $p^{(f)}$ is nonzero the magnetisation of the whole sample is given by

$$
\begin{aligned}
& m=(1-x)\left[p^{(f)} \bar{S} B_{\bar{S}}\left[\bar{S}\left(\lambda+\lambda^{\mathrm{ext}}\right)\right]+p^{(p)} \bar{S} B_{\bar{S}}\left[\bar{S} \lambda^{\mathrm{ext}}\right]\right] \\
& +x\left[p^{(f)} S B_{S}\left[S\left(\lambda+\lambda^{\mathrm{ext}}\right)\right]+p^{(p)} S B_{S}\left[S \lambda^{\mathrm{ext}}\right]\right] .
\end{aligned}
$$

\section{DC CONDUCTIVITY}

The above thermodynamic relations define the phase boundary between the ferromagnetic metallic and the paramagnetic insulating phase and explain the behaviour of the magnetisation and of the volume fractions of the different components. However, they do not contain any information about the resistivity of the system.

In the past, approaches which are based on the percolative mixing of regions with different macroscopic resistivities have been successfully used to fit experimental data $10,22.34$. Here we follow a similar path to model the resistivity $\rho$ of our mixed-phase system. Namely, we assume that the resistivity of the metallic component is proportional to the expression

$$
\begin{aligned}
\rho_{S}[z] & =\frac{g_{S}[z]-\gamma_{S}[z]^{2}}{\gamma_{S}[z]^{2}} \\
g_{S}[z] & =\frac{S B_{S}[z]}{(2 S+1)^{2}}\left[(2 S+2) \operatorname{coth} \frac{(S+1) z}{S}-\operatorname{coth} \frac{z}{2 S}\right] \\
& +\frac{S+1}{2 S+1},
\end{aligned}
$$

derived by Kubo and Ohata 31 , which associates $\rho$ with the fluctuation of the double-exchange matrix element caused by the thermal spin disorder. The resistivity of the insulating component is assumed to match the 


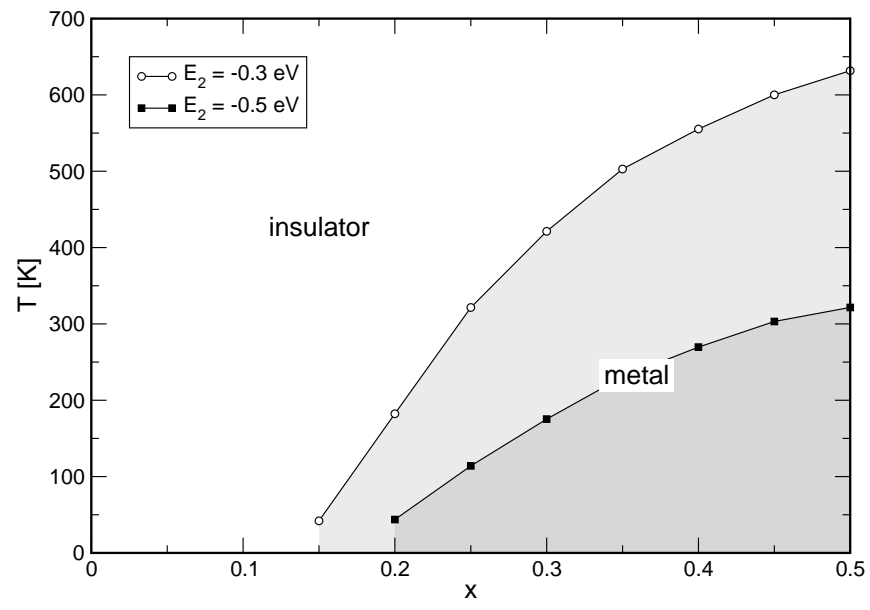

FIG. 2: $x$-T phase diagram of the mixed-phase model obtained for $W_{0}=2.4 \mathrm{eV}, E_{1}=-0.125 \mathrm{eV}$ and the two $E_{2}$ values $-0.3 \mathrm{eV}$ and $-0.5 \mathrm{eV}$.

resistivity of the high-temperature phase, which in experiment is well fit by the activated hopping of smallpolarons 5.7 .35 . Hence, the resistivities of the two components are given by,

$$
\begin{aligned}
& \rho^{(f)}=\frac{B}{x^{(f)}}\left(\rho_{S}\left[S\left(\lambda+\lambda^{\mathrm{ext}}\right)\right]+\rho_{\min }\right), \\
& \rho^{(p)}=\frac{A}{\beta x^{(p)}} \rho_{S}\left[S \lambda^{\mathrm{ext}}\right] e^{-\beta \epsilon_{p}},
\end{aligned}
$$

where the prefactors $A$ and $B$ as well as the cut-off $\rho_{\text {min }}$ are free model parameters which could be estimated from experimental data.

The resistivity of the whole sample, which may consist of an inhomogeneous mixture of both components, is calculated by assuming a random resistor network. More precisely, we choose nodes from a cubic lattice which belong to the metallic component with probability $p^{(f)}$ and to the polaronic component with probability $p^{(p)}$. Each of these nodes, which represent macroscopic regions of the sample, is connected to its neighbours with resistors of magnitude $\rho^{(f)}$ or $\rho^{(p)}$, respectively. The resistivity of the network then yields a reasonable approximation for the resistivity of the inhomogeneous ferromagnetic metallic phase of the manganites. The percolative nature of this model, particularly in the vicinity of the phase transition, makes the system very sensitive to small changes in temperature, doping and external magnetic field.

\section{RESULTS}

The numerical solution of the self-consistency equations is rather straightforward. However, some care is recommended, if there are multiple solutions for the equations (12)-15). To give an example, we set the bare band-width and the Jahn-Teller energy equal to
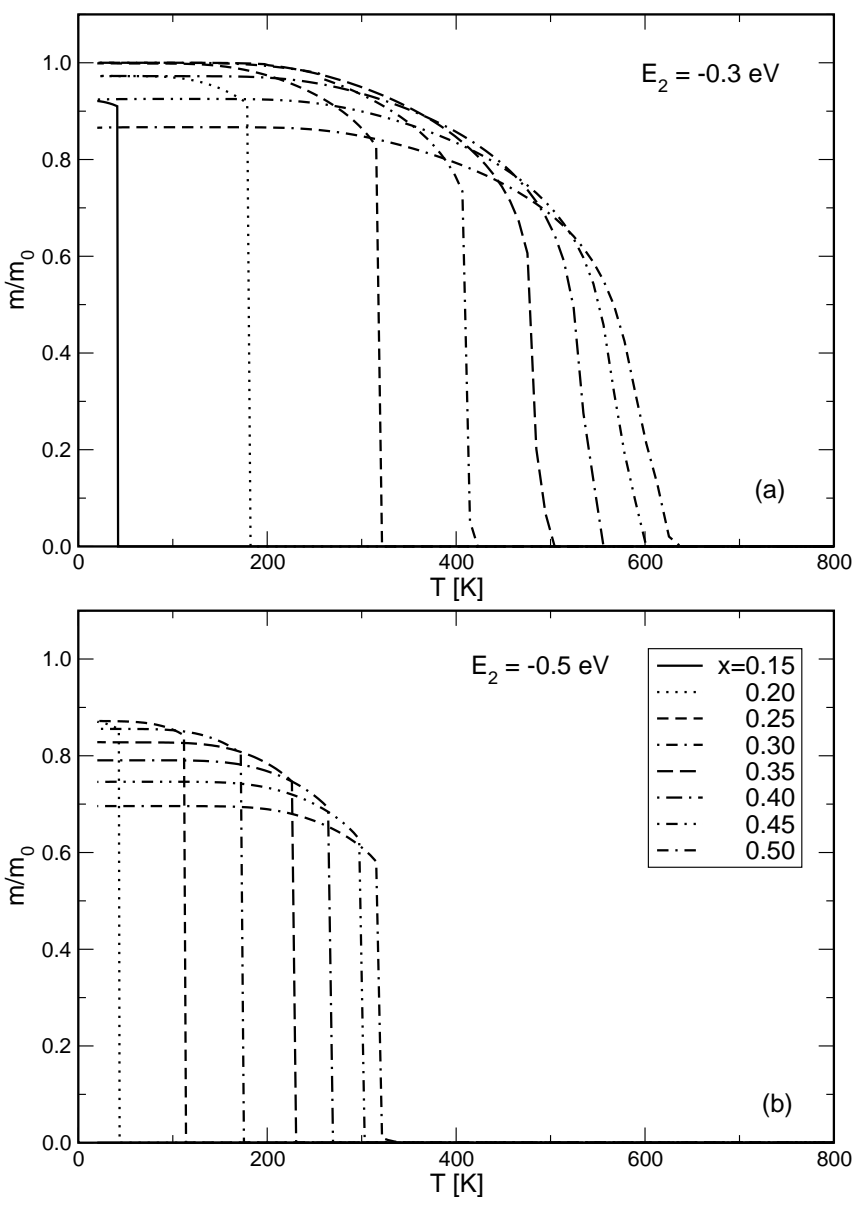

FIG. 3: Temperature dependence of the magnetisation $m$ for the two parameter sets and the indicated doping levels. Normalisation: $m_{0}=\bar{S}-x / 2$.

$W_{0}=2.4 \mathrm{eV}$ and $E_{1}=-0.125 \mathrm{eV}$, respectively, and consider two typical values for the polaronic binding energy, $E_{2}=-0.3 \mathrm{eV}$ and $-0.5 \mathrm{eV}$. This choice results in the phase boundaries displayed in Figure 2 Without an external magnetic field, for each doping $x$, the transition is defined by the critical temperature $T_{C}$ for which the magnetisation $m$ of the sample vanishes. The model yields reasonable values for both, the transition temperatures and the critical doping $x_{c}$ at $T=0$.

Figure 3 shows the magnetisation $m$ as a function of temperature and doping. Clearly, the order of the phase transition depends on both, the polaronic parameters $\left\{E_{1}, E_{2}\right\}$ and the doping $x$. Higher transition temperatures usually correspond to a continuous, second order transition, whereas otherwise the transition is first order. Similar behaviour was also found for the real materials 27,28 .

The inhomogeneous nature of the ferromagnetic and the paramagnetic phase becomes evident from Figure 4 where we show the dependence of the ferromagnetic volume fraction $p^{(f)}$ and of the corresponding carrier concentration $x^{(f)}$ on temperature and doping. In the case 


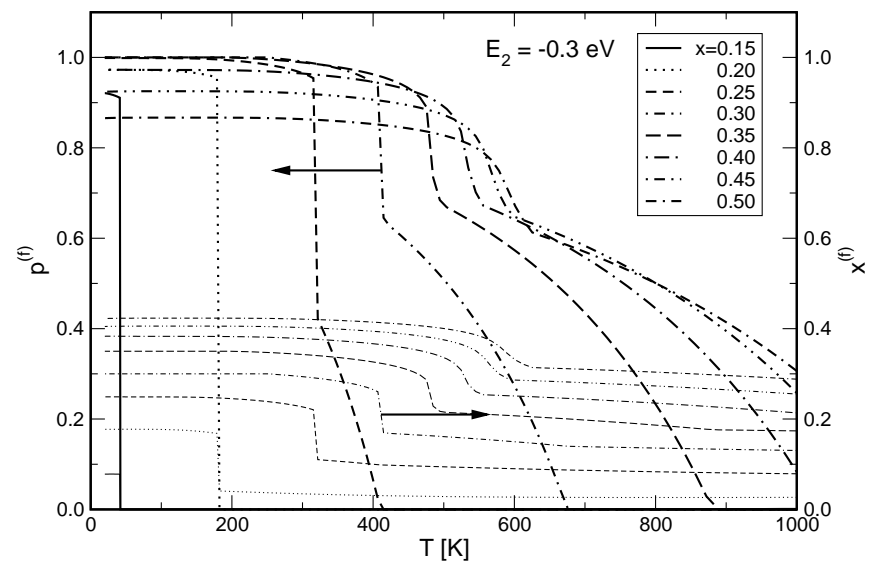

FIG. 4: Ferromagnetic volume fraction $p^{(f)}$ (bold lines) and corresponding carrier concentrations $x^{(f)}$ (thin lines) calculated with $E_{2}=-0.3 \mathrm{eV}$.

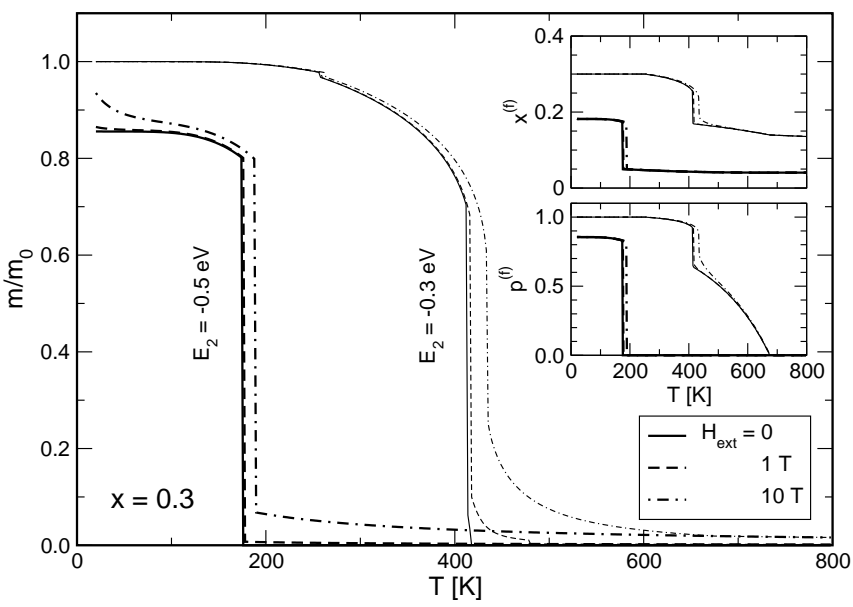

FIG. 5: Influence of an external magnetic field $H_{\text {ext }}^{z}$ on the magnetisation $m$ (main panel), the volume fraction $p^{(f)}$ and the carrier concentration $x^{(f)}$ (insets).

of small $T_{C}$ the sample is never completely metallic, i.e., a small polaronic insulating volume fraction is present even at lowest temperatures. On the other hand, for higher $T_{C}$ a finite metallic volume fraction exists also above $T_{C}$. Since it is usually smaller than the percolation threshold the sample remains insulating. In addition the carrier concentration within the metallic component is noticeable reduced above $T_{C}$. In the case of a second order transition $p^{(f)}$ and $x^{(f)}$ also decrease continuously with temperature. The presence of a metallic, doubleexchange driven component above $T_{C}$ can be related to the experimentally observed ferromagnetic correlations, sometimes interpreted as ferromagnetic clusters or magnetic polarons 36.37 .38 .39 .

The sensitivity of the phase transition and of all related quantities to an external magnetic field is illustrated in Figure [5. There we show the magnetisation
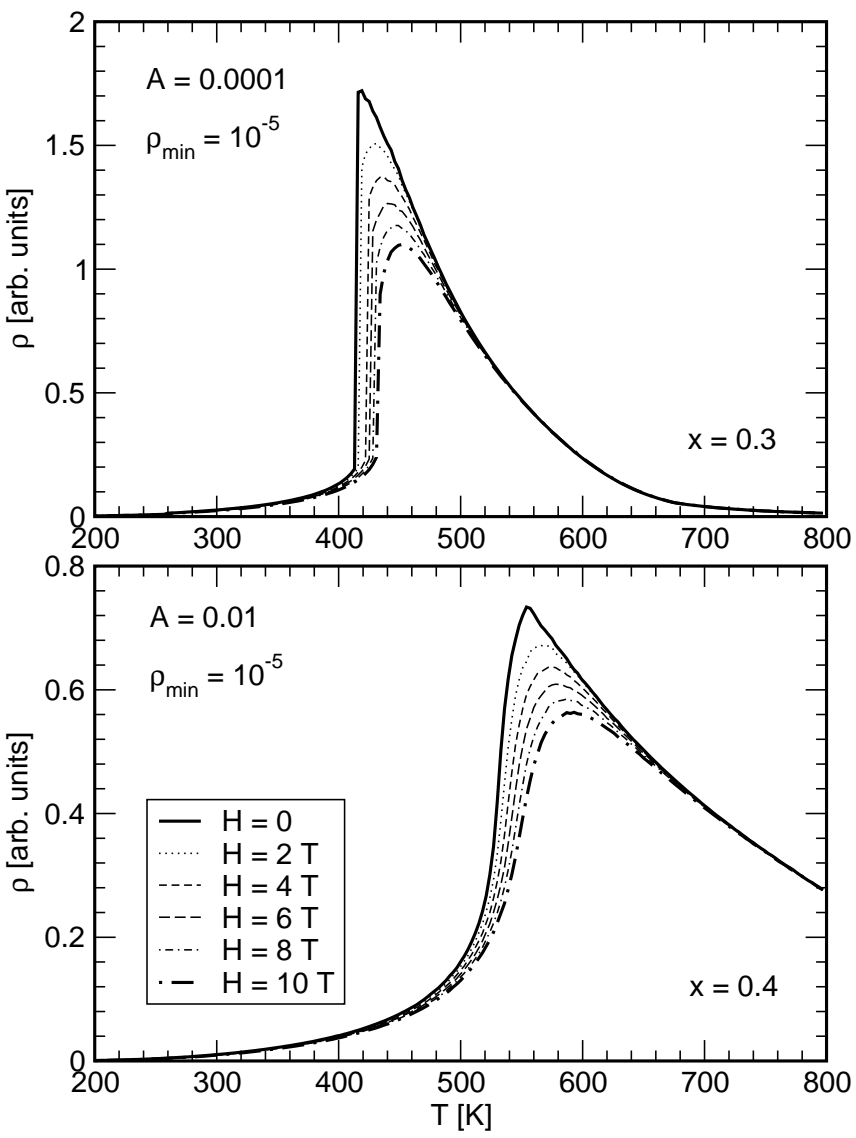

FIG. 6: Resistivity $\rho$ under the influence of an external magnetic field $H_{\text {ext }}^{z}$ for doping $x=0.3$ and 0.4 and the parameter set $\left\{W_{0}, E_{1}, E_{2}\right\}=\{2.4,-0.125,-0.3\} \mathrm{eV}$.

$m$ together with the volume fraction $p^{(f)}$ and the carrier concentration $x^{(f)}$ of the ferromagnetic metallic component for the two considered parameter sets and a doping level of $x=0.3$. Clearly, even for moderate field strength the critical temperature is shifted by a few degrees and in particular the volume fraction $p^{(f)}$ changes noticeable around $T_{C}$. Of course, the latter has an important influence on the conductivity of the system.

Inserting the volume fractions and carrier concentrations from the mixed-phase model into the ansatz for the DC conductivity we obtain the resistivities $\rho$ shown in Figure 6] Since we are mainly interested in the general features of $\rho$, we set $B=1$ and use the specified values for $A$ and $\rho_{\min }$. Depending on the order of the phase transition $\rho$ shows a sharp jump or a continuous increase close to $T_{C}$. This behaviour of the resistivity originates to a large degree from the changing volume fraction of the metallic component, which can cross the percolation threshold. However, the conductivity of the component itself as well as its carrier concentration strongly affect $\rho$ for $T<T_{C}$. An external magnetic field causes a reasonable suppression of $\rho$, i.e., a noticeable negative magnetoresistance. Compared to the real compounds the cal- 
culated effect is a bit weaker. Nevertheless, in view of the rather simple model for the conductivity the agreement is quite satisfactory. Probably, more involved assumptions for the resistivities of the two different components could improve the magnetoresistance data. At present the conductivity of the metallic component is controlled only by spin fluctuations in the double-exchange hopping, which could be increased by the weak anti-ferromagnetic interactions active in the manganites. In addition, other scattering mechanisms could play a role. The adiabatic small-polaron approximation used for the polaronic insulating volume fraction may be questionable as well, since the phonon modes involved in the electron-lattice coupling do not have small enough frequency. Another potential improvement concerns the site percolation model used to construct the random resistor network. As was pointed out recently, an approach that is based on correlated percolation could be more appropriate $\underline{40}$. An affinity to the formation of larger regions of the same type would naturally affect the resistivity of the system and its response to an external field.

\section{CONCLUSIONS}

In summary, we have proposed a phenomenological model for the ferromagnetic metallic phase of doped colossal magnetoresistive manganites, which is based on the coexistence of a double-exchange driven metallic component and a polaronic insulating component. Using modified equilibrium conditions and adding a percolative ansatz for the resistivity of the mixed phase we have substantially improved previous work ${ }^{26}$. With realistic parameters for the electronic band-width, the Jahn-Teller splitting and the polaronic binding energy our approach yields reasonable data for the phase boundary of the ferromagnetic metallic phase and correctly predicts the existence of a finite polaronic volume fraction well below the critical temperature $T_{C}$. The model shows a manifest sensitivity to external magnetic fields, including a large negative magnetoresistance close to $T_{C}$.

We thank F. Göhmann and N. Shannon for valuable discussions and acknowledge financial support by the Deutsche Forschungsgemeinschaft and the Czech Academy of Sciences under grant No. 436 TSE $113 / 33 / 0-2$.
1 A. P. Ramirez, J. Phys. Condens. Matter 9, 8171 (1997).

2 J. M. D. Coey, M. Viret, and S. von Molnár, Adv. Phys. 48, 167 (1999)

3 Y. Tokura and Y. Tomioka, J. Magn. Magn. Mater. 200, 1 (1999).

4 E. Dagotto, T. Hotta, and A. Moreo, Physics Reports 344, 1 (2001).

${ }^{5}$ M. Jaime, M. B. Salamon, M. Rubinstein, R. E. Treece, J. S. Horwitz, and D. B. Chrisey, Phys. Rev. B 54, 11914 (1996).

6 T. T. M. Palstra, A. P. Ramirez, S.-W. Cheong, B. R. Zegarski, P. Schiffer, and J. Zaanen, Phys. Rev. B 56, 5104 (1997).

7 D. C. Worledge, L. Miéville, and T. H. Geballe, Phys. Rev. B 57, 15267 (1998).

8 M. Jaime, H. T. Hardner, M. B. Salamon, M. Rubinstein, P. Dorsey, and D. Emin, Phys. Rev. Lett. 78, 951 (1997).

9 S. J. L. Billinge, R. G. DiFrancesco, G. H. Kwei, J. J. Neumeier, and J. D. Thompson, Phys. Rev. Lett. 77, 715 (1996).

10 M. Jaime, P. Lin, S. H. Chun, M. B. Salamon, P. Dorsey, and M. Rubinstein, Phys. Rev. B 60, 1028 (1999).

11 G. M. Zhao, V. Smolyaninova, W. Prellier, and H. Keller, Phys. Rev. Lett. 84, 6086 (2000).

12 R. H. Heffner, J. E. Sonier, D. E. MacLaughlin, G. J. Nieuwenhuys, G. Ehlers, F. Mezei, S.-W. Cheong, J. S. Gardner, and H. Röder, Phys. Rev. Lett. 85, 3285 (2000).

13 R. H. Heffner, J. E. Sonier, D. E. MacLaughlin, G. J. Nieuwenhuys, G. M. Luke, Y. J. Uemura, W. Ratcliff, S.W. Cheong, and G. Balakrishnan, Phys. Rev. B 63, 094408
(2001).

14 S. J. L. Billinge, T. Proffen, V. Petkov, J. L. Sarrao, and S. Kycia, Phys. Rev. B 62, 1203 (2000).

15 D. Louca, T. Egami, E. L. Brosha, H. Röder, and A. R. Bishop, Phys. Rev. B 56, R8475 (1997).

16 A. Lanzara, N. L. Saini, M. Brunelli, F. Natali, A. Bianconi, P. G. Radaelli, and S.-W. Cheong, Phys. Rev. Lett. 81, 878 (1998).

17 L. P. Gor'kov and V. Z. Kresin, JETP Lett. 67, 985 (1998).

18 L. P. Gor'kov and V. Z. Kresin, J. Supercond. 12, 243 (1999).

19 L. P. Gor'kov and V. Z. Kresin, J. Supercond. 13, 239 (2000).

20 A. Moreo, M. Mayr, A. Feiguin, S. Yunoki, and E. Dagotto, Phys. Rev. Lett. 84, 5568 (2000).

21 M. Fäth, S. Freisem, A. A. Menovsky, Y. Tomioka, J. Aarts, and J. A. Mydosh, Science 285, 1540 (1999).

22 T. Becker, C. Streng, Y. Luo, V. Moshnyaga, B. Damaschke, N. Shannon, and K. Samwer, Phys. Rev. Lett. 89, 237203 (2002).

23 M. Uehara, S. Mori, C. H. Chen, and S.-W. Cheong, Nature 399, 560 (1999).

24 P. G. Radaelli, R. M. Ibberson, D. N. Argyriou, H. Casalta, K. H. Andersen, S.-W. Cheong, and J. F. Mitchell, Phys. Rev. B 63, 172419 (2001).

${ }^{25}$ C. Simon, S. Mercone, N. Guiblin, C. Martin, A. Brûlet, and G. André, Phys. Rev. Lett. 89, 207202 (2002).

26 A. Weiße, J. Loos, and H. Fehske, Phys. Rev. B 64, 104413 (2001).

27 J. Mira, J. Rivas, F. Rivadulla, C. Vázquez-Vázquez, and 
M. A. López-Quintela, Phys. Rev. B 60, 2998 (1999).

28 D. Kim, B. Revaz, B. L. Zink, F. Hellman, J. J. Rhyne, and J. F. Mitchell, Phys. Rev. Lett. 89, 227202 (2002).

29 C. Zener, Phys. Rev. 82, 403 (1951).

30 P. W. Anderson and H. Hasegawa, Phys. Rev. 100, 675 (1955).

31 K. Kubo and N. Ohata, J. Phys. Soc. Jpn. 33, 21 (1972).

32 P. W. Anderson, Phys. Rev. 115, 2 (1959).

33 K. I. Kugel and D. I. Khomskii, JETP Lett. 15, 446 (1972).

34 A. N. Lagarkov and A. K. Sarychev, Phys. Rev. B 53, 6318 (1996).

35 D. Emin and T. Holstein, Ann. Phys. (N.Y.) 53, 439 (1969).
36 J. de Teresa, M. R. Ibarra, J. Blasco, J. García, C. Marquina, P. A. Algarabel, Z. Arnold, K. Kamenev, C. Ritter, and R. von Helmolt, Phys. Rev. B 54, 1187 (1996).

37 V. Chechersky, A. Nath, I. Isaac, J. P. Franck, K. Ghosh, H. Ju, and R. L. Greene, Phys. Rev. B 59, 497 (1999).

38 D. E. Cox, P. G. Radaelli, M. Marezio, and S.-W. Cheong, Phys. Rev. B 57, 3305 (1998).

39 I. G. Deac, J. F. Mitchell, and P. Schiffer, Phys. Rev. B 63, 172408 (2001).

40 L. Khomskii and D. Khomskii (2002), URL http://arXiv.org/abs/cond-mat/0210616 